\title{
On the upper limit of thermal conductivity GaN crystals
}

\author{
B A Danilchenko ${ }^{\text {a,* }}$ I A Obukhov ${ }^{\text {a }}$ T Paszkiewicz $^{\text {b }}$ S Wolski ${ }^{\text {b }}$ \\ A. Jeżowski ${ }^{\mathrm{c}}$ \\ ${ }^{a}$ Institute of Physics, National Academy of Sciences of Ukraine, Prospect Nauki 46, \\ 252650 Kiev, Ukraine \\ ${ }^{\mathrm{b}}$ Faculty of Mathematic and Applied Physics, Rzeszów University of Technology, ul. W. \\ Pola 2, PL-35-959 Rzeszów Poland \\ ${ }^{\mathrm{c}}$ Institute of Low Temperature and Structure Reasearch, Polish Academy of Sciences, P.O. \\ Box 1410, 50-422 Wroctaw, Poland
}

\begin{abstract}
The maximal value of thermal conductivity $\kappa_{\max }$ of the perfect wurzite GaN crystal containing isotopes of natural abundance is estimated. Our upper limit of $\kappa=4800 \mathrm{~W} / \mathrm{Km}$ at $T_{\max }=32 \mathrm{~K}$ is smaller than calculated by Liu and Balandin $\kappa=6000 \mathrm{~W} / \mathrm{Km}$ and higher than obtained by Slack et al $\kappa=3750 \mathrm{~W} / \mathrm{Km}$. The phenomenological dependence $\kappa \propto T^{-1.43}$ obtained by Mion et al for the temperature interval $300-450 \mathrm{~K}$ is extended to $200-300 \mathrm{~K}$. For temperatures higher than $T_{\max }$ the best fitting of our experimental data to Callaway's formula is obtained for Grueneisen's constant equal to $\gamma=1.35$.
\end{abstract}

Key words: semiconductors, point defects, heat conduction

PACS: 66.70.+f, 71.55.-i

In heterostructures GaN/AlGaN in electric field of the order of $100 \mathrm{kV} / \mathrm{cm}$, the density of dissipated power reaches $10^{6} \mathrm{~W} / \mathrm{cm}^{2}[1 / 2 \mid 3]$. This means that gallium nitride based devices suffer from heating effects that significantly limit the performance of high electron mobility transistors and laser diodes thereby reducing the device's life and reliability. This means that the accurate knowledge of the gallium nitride thermal conductivity is crucial to understanding thermal effects. For this reason,

\footnotetext{
* Corresponding author

Email address: danil@iop.kiev.ua (B A Danilchenko).
} 
the investigation of the temperature behavior of thermal conductivity coefficient is an actual and reasonable problem.

Recently four groups measured thermal conductivity of $\mathrm{GaN}$ in the wide temperature ranges. Luo et al [4] have measured the thermal conductivity of both LEO and HVPE single crystals of GaN over the temperature range $60-300 \mathrm{~K}$. Slack et al [56] performed measurements in the temperature interval $11-300 \mathrm{~K}$. We measured thermal conductivity of massive high pressure growth dislocationfree crystalline sample containing about $10^{20}$ per $\mathrm{cm}^{-3}$ point defects in the temperature interval $4.5-300 \mathrm{~K}$ and performed a preliminary analysis of temperature dependence of it [7]. We also measured heat capacity of $\mathrm{GaN}$ for temperatures between $5 \mathrm{~K}$ and $300 \mathrm{~K}$, and as a result we established the value of Debye's temperature of $\mathrm{GaN}$ [8]. These measurements also allow us to obtain the temperature dependence of the phonon mean free path $L_{\text {eff }}$. Measurements of thermal conductivity for temperatures higher than $300 \mathrm{~K}$ were performed by Mion et al [9].

Slack et al compared their experimental results to the Callaway formula considering separately the contributions of longitudinal and transverse phonons. Therefore, their fitting procedure relays on six fitting parameters, namely two Debye's temperatures $\theta_{\mathrm{L}}, \theta_{\mathrm{T}}$ Grueneisen's constants $\gamma_{\mathrm{L}}, \gamma_{\mathrm{T}}$ and constants $b_{\mathrm{L}}, b_{\mathrm{T}}$ related to the Umklapp processes ( $\mathrm{L}$ and $\mathrm{T}$ stands for longitudinal and transverse phonons, respectively). The single set of their free adjustable parameters contained also the relaxation rate for the normal processes $\tau_{N}^{-1}$. Liu et Balandin [10] considered thermal conductivity GaN. They argued that in the case of $\mathrm{GaN}$ specimens the normal processes do not influence the thermal conductivity because of strong scattering on point defects. For this reason we use Calaway's formula with $\kappa_{2}=0$. The results of measurements of thermal conductivity on wurzite GaN samples [4567] were analyzed by Kamatagi et al [11] employing Holland's and modified Callaway's models. A good representation of the temperature dependence of $\kappa$ was obtained with a single set of phonon-phonon scattering prameters. The purpose of this study is different than Kamatagi et al [11]. Having in mind the needs of high-power electronic devices manufactured from $\mathrm{GaN}$ we shall establish the upper limit of thermal conductivity for $T>T_{\max }$. We base our approach on the standard Callaway formula and use only two free adjustable parameters.

In dielectric and semiconducting crystals heat is carried mainly by acoustic phonons. As a rule, the group velocities of such phonons are much greater than those of optical phonons. Therefore, heat flow in semiconducting crystals is limited by various mechanisms of scattering of acoustic phonons. There are two principal kinds of phonon scattering in crystals, namely resistive and normal processes [12]. They are characterized by the corresponding relaxation times $\tau_{\mathrm{R}}$ and $\tau_{\mathrm{N}}$. Normal processes do not change the total momentum of phonons. Such processes influence the heat flow only indirectly. The resulting phonons of higher energy are scattered more intensively on point defects and have greater probability of participating in Umklapp processes. In the perfect crystals (dislocation and chemical impurity-free 
samples), the relevant intrinsic resistive scattering is due to three-phonon Umklapp with relaxation time $\tau_{\mathrm{U}}$, the point defect scattering with characteristic time $\tau_{\mathrm{P}}$, and the boundary scattering $\tau_{\mathrm{B}}$. The characteristic time $\tau_{\mathrm{P}}$ is determined mostly by the crystal natural isotope composition.

The total thermal conductivity $\kappa(T)$ can be written as [13]

$$
\kappa=\kappa_{1}+\kappa_{2}
$$

where $\kappa_{1}(T)$ and $\kappa_{2}(T)$ are defined by

$$
\begin{gathered}
\kappa_{1}(T)=\left(\frac{k_{B}}{\hbar}\right)^{3} \frac{k_{B}}{2 \pi^{2} \bar{v}} T^{3} \int_{0}^{\frac{\theta_{D}}{T}} \frac{\tau_{c} x^{4} e^{x}}{\left(e^{x}-1\right)^{2}} d x \\
\kappa_{2}(T)=\left(\frac{k_{B}}{\hbar}\right)^{3} \frac{k_{B}}{2 \pi^{2} \bar{v}} T^{3}\left\{\int_{0}^{\frac{\theta_{D}}{T}} \frac{\tau_{C}}{\tau_{N}} x^{4} e^{x}\left(e^{x}-1\right)^{-2} d x\right\}\left[\int_{0}^{2} \frac{\tau_{C}}{\tau_{N} \tau_{R}} x^{4} e^{x}\left(e^{x}-1\right)^{-2} d x\right]^{-1},
\end{gathered}
$$

where $\mathrm{k}_{\mathrm{B}}$ and $\hbar$ are Boltzmann's and Planck's constants respectively, $\bar{v}$ is the mean velocity of phonons, $\theta_{D}$ is Debye's temperature, $T$ is the ambient temperature, $x=$ $\hbar \omega / k_{B} T, \omega$ is the phonon frequency, $\tau_{C}^{-1}=\tau_{R}^{-1}+\tau_{N}^{-1}$, and $\tau_{R}^{-1}=\tau_{U}^{-1}+\tau_{P}^{-1}+\tau_{B}^{-1}$.

When $\tau_{N}>\tau_{R}$, the combined relaxation time $\tau_{c} \approx \tau_{R}$ and the term $\kappa_{2}(3)$ is negligibly small. The generally accepted expression for Umklapp phonon scattering rate is (cf. e.g. [5])

$$
\tau_{U}^{-1}=\frac{\hbar \gamma^{2} T \omega^{2}}{M \bar{v}^{2} \theta_{D}} \exp \left(-\frac{\theta_{D}}{b T}\right),
$$

where $\gamma$ is Grueneisen's constant, $M$ is the mass of GaN molecule, and is $b$ is a fitting parameter. As a rule for crystalline solids, $2 \leq b \leq 3$ [12].

For phonons scattered by isolated defects of mass different from that of the host in an otherwise perfect crystal, Tamura derived a scattering rate [14]

$$
\left[\tau_{P}(x)\right]^{-1}=\frac{V k_{B}^{4} \tilde{g}}{12 \pi \hbar^{4} \bar{v}^{3}} x^{4} T^{4}
$$

where $V$ is the volume of the elementary cell, the parameter $\tilde{g}$ is

$$
\tilde{g}=\frac{\sum_{\sigma} g(\sigma) \bar{M}^{2}(\sigma)}{\left[\sum_{\sigma^{\prime}} \bar{M}\left(\sigma^{\prime}\right)\right]^{2}}
$$

with $g(\sigma)$ is the constant which express the strength of isotope effecs. It depends 
on mass $M_{i}(\sigma)$ and fraction $f_{i}(\sigma)$ in the ith isotope of $\sigma$ atom as

$$
g(\sigma)=\sum_{i} f_{i}(\sigma)\left[1-\frac{M_{i}(\sigma)}{\bar{M}(\sigma)}\right]^{2} .
$$

Above $\bar{M}(\sigma)=\sum_{i} f_{i}(\sigma) M_{i}(\sigma)$ is the average mass of $\sigma$ atom.

The boundary scattering is important for low temperatures and does not influence thermal conductivity in the intermediate and high temperatures. The related scattering rate $\tau_{B}^{-1}$ is

$$
\tau_{B}^{-1}=\frac{\bar{v}}{L_{\text {eff }}}
$$

where $L_{\text {eff }}$ is the effective phonon mean free path. For $T \ll \theta_{\mathrm{D}}$ it is of the order of the cross-sectional dimensions. A precise determination of $L_{\text {eff }}$ is crucial for correctly describing the $\kappa(T)$ curve in the low temperature range.

As we have recently established, for $\mathrm{GaN}$, the Debye temperature $\theta=365 \mathrm{~K}[\overline{8}]$, which is lower than used by other authors [5|6|10|15|16]. It is therefore obvious that one should revise the fitting procedure. We calculate $\tilde{g}(\mathrm{Eq} 6$ accounting for natural composition of isotopes of $\mathrm{Ga}$ and $\mathrm{N}$. Our calculations show, in agreement with ref. [6], that the contribution of nitrogen isotopes to $\tilde{g}$ is ten times smaller than the contribution of isotopes of gallium.

Since other defects always present in available specimens diminish the thermal conductivity coefficient, we obtain the upper limit of thermal conductivity of GaN specimens with the natural composition of isotopes. The Grueneisen parameter for hexagonal GaN was calculated by Łopuszyński and Majewski [17]. Their value $\gamma=1.28$ is bigger that used by other authors [6 10[18]. For temperatures higher than $T_{\max }$ (corresponding to $\kappa_{\max }$ ), the best fitting is obtained for $\gamma=1.35$, which is close to the calculated value [17]. As a result of fitting the Callaway formula to our experimental results (with $\tau_{N} \gg \tau_{R}$ ), we found $b=2.5$. In Table 1 we collected the values of the above parameters.

\begin{tabular}{|c|c|c|c|c|}
\hline $\bar{v}[\mathrm{~cm} / \mathrm{s}]$ & $\gamma$ & $\tilde{g}$ & $b$ & $\theta_{\mathrm{D}}[\mathrm{K}]$ \\
\hline $5.1 \times 10^{5}$ & 1.35 & $1.37 \times 10^{-4}$ & 2.5 & 365 \\
\hline
\end{tabular}

Table 1

Single parameter set based on Callaway's model used to represent the temperature dependence of the thermal conductivity $\kappa$

Extension of temperature dependence calculated by us is in excellent agreement with $\kappa \propto T^{-1.43}$ obtained in ref. [9] in the temperature interval $300-450 \mathrm{~K}$ for a specimen containing dislocations of the lowest density. This means that the phenomenological expression $\kappa=\kappa(300 \mathrm{~K}) \times(T / 300 \mathrm{~K})^{-1.43}$ proposed in ref. [19] can be also used in temperature interval $200-450 \mathrm{~K}$. One should stress that this phenomenological expression differs from the results set forth in ref. [6] (cf. Fig. 11). The observed numerical values of $\kappa$ and the temperature slope of $\kappa(T)$ indicates 
that $3 \omega$ dynamic method of thermal conductivity measurements [9] and the axial stationary heat flow method measurements [7] yield the same temperature dependence, hence these two experimental techniques should be considered as supplementary.

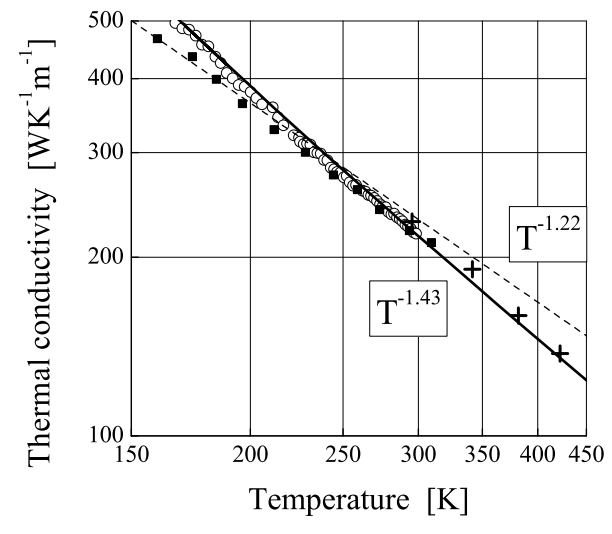

Fig. 1. Thermal conductivity $\kappa(T)$ of $\mathrm{GaN}$ for $T>T_{\max }$. Open circles - our results [7], crosses are taken from ref. [9] for sample with the low dislocations density, filled squares ref. [5]. Solid line - the present calculations, dotted line $-\kappa \propto T^{-1.22}$ ref. [5].

In Fig. 2 we present the results of our calculations for $3 \leq T \leq 500 \mathrm{~K}$ [7] and compare them with the results of calculations presented in refs. [6 9]10]. In the low temperature limit, where the boundary scattering is essential, the calculated $\kappa$ was fitted to our experimental results using the value of $L_{\text {eff }}=0.08 \mathrm{~cm}$. This value is close to the low temperature mean free path of acoustic phonons reported in ref. [8].

The mean value of velocity is calculated using the set of elastic constants reported by Polian et al [20]. The obtained value of $\bar{v}$ is close to velocity $\bar{v}=6 \times 10^{5} \mathrm{~cm} / \mathrm{s}$ measured in our experiments on propagation of phonon pulses at helium temperatures [21].

Our upper limit of $\kappa\left(\kappa_{\max }=4800 \mathrm{~W} / \mathrm{Km}\right.$ at $\left.T_{\max }=32 \mathrm{~K}\right)$ is smaller than calculated by Liu and Balandin $\kappa_{\max }=6000 \mathrm{~W} / \mathrm{Km}$ [10] and higher than value $\kappa_{\max }=3750 \mathrm{~W} / \mathrm{Km}$ announced in ref. [6].

In conclusion, for hexagonal GaN crystals containing isotope atoms and otherwise perfect crystal, we obtained an acceptable representation of all our data for temperatures between $3 \mathrm{~K}$ and $500 \mathrm{~K}$. Using Callaway's model with $\kappa_{2}=0\left(\tau_{\mathrm{N}} \gg \tau_{\mathrm{R}}\right)$, we adjusted two free parameters $(\gamma$ and $b)$ in the combination of the two scattering mechanisms considered, with the scattering rates for isotopic and boundary scattering given in Eqs. (5) - (8). The remaining parameters were calculated or deduced from independent experiments. We established that thermal conductivity for tem- 


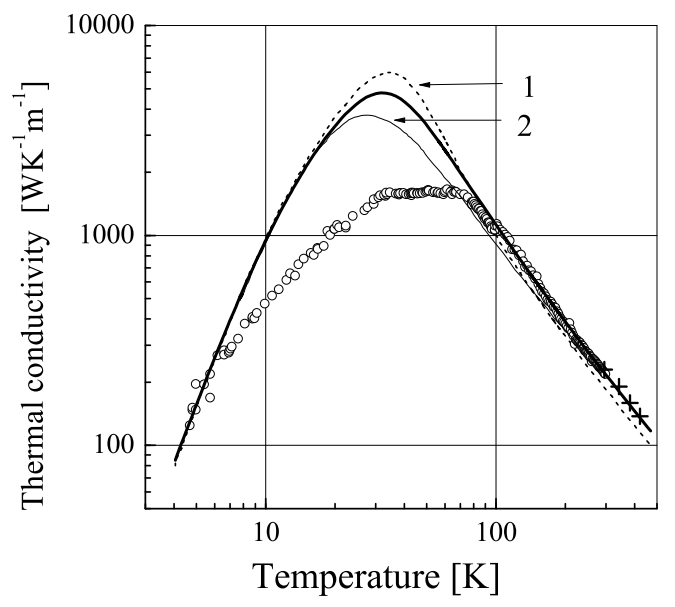

Fig. 2. Thermal conductivity of gallium nitride in the temperature interval $5-450 \mathrm{~K}$. Open circles - results for our best samples [7], thick solid line - present calculation, crosses ref. [9], curve No. 1 - ref. [10], curve No. 2 - ref. [6].

peratures greater than $T_{\max }$ is determined by scattering by point mass defects and the Umklapp processes. In the temperature range $200-500 \mathrm{~K}$ we justified the empirical dependence $\kappa \propto T^{-1.43}$ observed by Mion et al [9].

\section{Acknowledgments}

This work was supported by the Program of Targeted Initiatives established by STCU USA and NASU Ukraine under Grant No. 3922.

\section{References}

[1] B. A. Danilchenko, S. E. Zelensky, E. Drok, S. A. Vitusevich, N. Danylyuk, S. V. Klein, H. Lüth, A. E. Belyaev, V. A. Kochelap, Appl. Phys. Lett. 85 (2004) 5421.

[2] J. Kuzmík, S. Bychikhin, D. Pogany, C. Gaquiere, E. Morvan, J. Appl. Phys. 99 (2006) 123720.

[3] J. M. Barker, D. K. Ferry, S. M. Goodnick, D. D. Koleske, A. Allerman, R. J. Shul, J. Vac. Sci. Technol. 22 (2004) 2045.

[4] C. Luo, D. R. Clarke, J. R. Dryden, J. Electron. Mater. 30 (2001) 138.

[5] G. A. Slack, L. J. Schowalter, D. Morelli, J. A. Freita Jr, J.Cryst. Growth. 246 (2002) 287. 
[6] D. T. Morelli, J. P. Heremans, G. A. Slack, Phys. Rev. B 66 (2002) 195304.

[7] A. Jeżowski, B. Danilchenko, M. Boćkowski, I. Grzegory, S. Krukowski, T. Suski, T. Paszkiewicz, Solid State Com. 128 (2003) 69.

[8] B. A. Danilchenko, T. Paszkiewicz, S. Wolski, A. Jeżowski, T. Plackowski, Appl. Phys. Lett. 89 (2006) 061901.

[9] C. Mion, J. F. Muth, E. A. Preble, D. Hanser, Appl. Phys. Lett. 89 (2006) 092123.

[10] W. Liu, A. A. Balandin, J. Appl. Phys. 97 (2005) 073710.

[11] M. Kamatagi, N. Sankeshwar, B. Mulimani, Diamond \& Related Materials 16 (2007) 98.

[12] R. Berman, Thermal Conductivity in Solid, Oxford University Press, Oxford, 1976.

[13] J. Callaway, Phys. Rev. 113 (1959) 1046.

[14] S. I. Tamura, Phys. Rev. 30 (1984) 849.

[15] J. Zou, D. Kotchetkov, A. A. Balandin, D. I. Florescu, F. H. Pollak, J. Appl. Phys. 92 (2002) 2534.

[16] D. Kotchetkov, J. Zou, A. A. Balandin, D. I. Florescu, F. H. Pollak, Appl. Phys. Lett. 79 (2001) 4316.

[17] M. Łopuszyński, J. Majewski, Ab initio calculations of third-order elastic constants and related properties for selected semiconductors, arXiv:cond-mat/0701410v2 (Apr 2007).

[18] H. Iwanag, A. Kunishig, S. Takeuchi, J. Mat. Sc. 35 (2000) 2451.

[19] C. Mion, Investigation of the thermal properties of gallium nitride using the three omega technique, Ph.D. thesis, North Carolina State University Raleigh (2005).

[20] A. Polian, M. Grimsditch, I. Grzegory, J. Appl. Phys 79 (1996) 3343.

[21] B. A. Danilchenko, M. Boćkowski, I. Grzegory, V. Guzenko, T. Paszkiewicz, T. Suski, Physica B 263-264 (1999) 727. 